\title{
WEIGHTED INEQUALITIES AND VECTOR-VALUED CALDERÓN-ZYGMUND OPERATORS ON NON-HOMOGENEOUS SPACES
}

\author{
J. García-Cuerva and J. M. Martell
}

\begin{abstract}
Recently, F. Nazarov, S. Treil and A. Volberg (and independently $\mathrm{X}$. Tolsa) have extended the classical theory of Calderón-Zygmund operators to the context of a "non-homogeneous" space $(\mathbb{X}, d, \mu)$, where, in particular, the measure $\mu$ may be non-doubling. In the present work we study weighted inequalities for these operators. Specifically, for $1<p<\infty$, we identify sufficient conditions for the weight on one side, which guarantee the existence of another weight in the other side, so that the weighted $L^{p}$ inequality holds. We deal with this problem by developing a vector-valued theory for Calderón-Zygmund operators on non-homogeneous spaces which is interesting in its own right. For the case of the Cauchy integral operator, which is the most important example, we even prove that the conditions for the weights are also necessary.
\end{abstract}

\section{Introduction}

Let $\mu$ be a Borel measure in the complex plane. The Cauchy integral operator is defined as

$$
\mathcal{C} f(z)=\mathcal{C}_{\mu} f(z)=\int_{\mathbb{C}} \frac{f(\xi)}{z-\xi} d \mu(\xi), \quad \text { for } \mu \text {-a.e. } z \in \mathbb{C} \backslash \operatorname{supp} f .
$$

It is natural to wonder whether this operator is bounded on $L^{2}(\mu)$, on $L^{p}(\mu)$ or even between $L^{1}(\mu)$ and $L^{1, \infty}(\mu)$. Besides, since the previous definition makes no sense for points in the support of the function,

2000 Mathematics Subject Classification. 42B20, 30E20.

Key words. Non-doubling measures, Calderón-Zygmund operators, vector-valued inequalities, weights, Cauchy integral.

Both authors are partially supported by DGES Spain, under Grant PB97-0030.

We would like to thank J. L. Torrea for many comments and suggestions. 
another question is to find conditions on $\mu$ in order to ensure the existence of the principal values on these spaces. For example, when $\mu$ is the one-dimensional Hausdorff measure over a Lipschitz curve, the boundedness was proved in $[\mathbf{C a l}]$ for small Lipschitz constant and the full result was obtained in $[\mathbf{C M M}]$. Another approach to this problem is the $T(b)$ theorem proved in [DJS], (see also [Da1]). For measures over rectifiable sets and the relation with analytic capacity see [Ch1], [Ch2], [Mur] and the references given there. See also the recent survey [Da2]. The answer for general measures has been obtained by Tolsa in $[$ To1 $],[$ To2 $]$. In the first work, it is established the equivalence of the uniform boundedness of the truncated Cauchy integrals in $L^{2}(\mu)$ and some geometric conditions on the measure $\mu$, namely: $\mu$ has linear growth - which means that the measure of each ball is controlled by a constat times the radius - and it satisfies certain local curvature condition (see $[\mathbf{T o 1}],[\mathbf{M e l}],[\mathbf{M V}],[\mathbf{M M V}]$ ). In the second reference, the author obtains that the boundedness in $L^{2}(\mu)$ implies the existence of principal values. Besides, those measures, for which the existence of principal values holds, are completely characterized.

In [NTV1] a $T(1)$ theorem is proved for Calderón-Zygmund operators in $\mathbb{C}$ with a measure such that $\mu(Q) \leq \ell(Q)$ for all squares $Q \subset \mathbb{C}$, where $\ell(Q)$ stands for the side length of $Q$. They prove that $T$ is continuous in $L^{2}(\mu)$ if and only if $T$ and its adjoint are bounded over characteristic functions of squares. (Actually, as it is pointed out in [NTV1], similar results work for " $n$-dimensional" measures in $\mathbb{R}^{d}, d \geq n$ and CalderónZygmund operators with " $n$-dimensional" kernels $\bar{K}$.) In particular for the Cauchy integral, this result has been also obtained by [Ver]. In [NTV2] a generalization of this setting is given. They deal with nonhomogeneous spaces which are metric spaces endowed with a positive measure in such a way that the measure of a ball is controlled by the radius to the power $n$, where $n>0$ is a fixed real number. In these spaces (where the measure is not assumed to satisfy any doubling condition) from the $L^{2}(\mu)$ boundedness, the authors manage to obtain weak and strong type estimates for Calderón-Zygmund operators and for the maximal operators associated with them. The main example is the Cauchy integral where the metric space is $\mathbb{C}$ and $n=1$.

A non-homogeneous space $(\mathbb{X}, d)$ will be a separable metric space endowed with a non-negative " $n$-dimensional" Borel measure $\mu$, that is,

$$
\mu(B(x, r)) \leq r^{n}, \quad \text { for all } x \in \mathbb{X}, r>0,
$$

where $B(x, r)=\{y \in \mathbb{X}: d(x, y) \leq r\}$ and $n$ is a fixed positive number (not necessarily an integer). 
Definition 1.1. A bounded linear operator $T$ on $L^{2}(\mu)$ is said to be a Calderón-Zygmund operator with " $n$-dimensional" kernel $K$ if for every $f \in L^{2}(\mu)$,

$$
T f(x)=\int_{\mathbb{X}} K(x, y) f(y) d \mu(y), \quad \text { for } \mu \text {-almost every } x \in \mathbb{X} \backslash \operatorname{supp} f,
$$

where, for some $A>0, K: \mathbb{X} \times \mathbb{X} \longrightarrow \mathbb{C}$ satisfies

(i) $|K(x, y)| \leq \frac{A}{d(x, y)^{n}}$, for all $x \neq y$;

(ii) and the following two conditions hold:

$$
\begin{aligned}
& \int_{d(x, y) \geq 2 d\left(x, x^{\prime}\right)}\left|K(x, y)-K\left(x^{\prime}, y\right)\right| d \mu(y) \leq A \\
& \int_{d(x, y) \geq 2 d\left(x, x^{\prime}\right)}\left|K(y, x)-K\left(y, x^{\prime}\right)\right| d \mu(y) \leq A .
\end{aligned}
$$

Note that this class of operators is slightly larger than those considered in [NTV2] where pointwise estimates for the kernel are used rather than integral estimates. Observe that if we take some measure $\mu$ in $\mathbb{C}$ such that the Cauchy integral is bounded in $L^{2}(\mu), \mu$ will have linear growth (e.g. [To1]), that is, $\mu$ is "1-dimensional". In this case, the Cauchy integral is a Calderón-Zygmund operator with "1-dimensional" kernel $K(z, \xi)=\frac{1}{z-\xi}$.

The aim of this paper is to obtain some weighted inequalities for these operators. If $1<p<\infty$, consider the following two-weight inequality for $T$ :

$$
\int_{\mathbb{X}}|T f(x)|^{p} u(x) d \mu(x) \leq \int_{\mathbb{X}}|f(x)|^{p} v(x) d \mu(x),
$$

for $f \in L^{p}(v)=L^{p}(v d \mu)$ and where $u, v$ are $\mu$-a.e. positive functions.

These inequalities in $\mathbb{R}^{d}$ with the same weight in both sides have been recently studied by $[\mathbf{O P}]$. They have obtained some results about Muckenhoupt weights and weighted inequalities for Calderón-Zygmund operators. However, we are interested in a different type of inequalities, namely, we shall be concerned with the following problem:

Find conditions on $0 \leq v<\infty \mu$-a.e. (resp. $u>0$ $\mu$-a.e.) such that (1) is satisfied by some $u>0 \mu$-a.e. (resp. $0 \leq v<\infty \mu$-a.e.). 
As we can see in Chapter VI of [GR] and in Chapters II, V of [Ste] this question is closely related to obtaining vector-valued inequalities for $T$. We shall use this connection to get an answer for this problem, that is, we shall develop a vector-valued theory for these operators to obtain the necessary vector-valued inequalities. Some references about classical vector-valued theory are $[\mathbf{B C P}],[\mathbf{R R T}]$ and $[\mathbf{G R}]$.

For these operators the relevant classes of weights will be, as usual, $D_{p}$ and $Z_{p}, 1<p<\infty$, which are defined as follows:

$$
\begin{aligned}
D_{p} & =\left\{0 \leq w<\infty \mu \text {-a.e. }: \int_{\mathbb{X}} w(x)^{1-p^{\prime}}\left(1+d\left(x, x_{0}\right)\right)^{-n p^{\prime}} d \mu(x)<\infty\right\} \\
Z_{p} & =\left\{w>0 \mu \text {-a.e. : } \int_{\mathbb{X}} w(x)\left(1+d\left(x, x_{0}\right)\right)^{-n p} d \mu(x)<\infty\right\},
\end{aligned}
$$

for some $x_{0} \in \mathbb{X}$. Note that these classes of weights do not depend on the point $x_{0}$ and that this definition becomes simpler for spaces with finite diameter (see Section 3). The concrete result is

Theorem. Take $p, 1<p<\infty$. If $u \in Z_{p}$ (resp. $v \in D_{p}$ ), then there exists some weight $0<v<\infty \mu$-a.e. (resp. $0<u<\infty \mu$-a.e.) such that (1) holds. Moreover, $v$ (resp. u) can be found in such a way that $v^{\alpha} \in Z_{p}$ (resp. $u^{\alpha} \in D_{p}$ ), provided that $0<\alpha<1$.

Once we have obtained sufficient conditions on the weights in order to ensure that (1) holds, we shall study how sharp are these classes, that is, we shall prove that for a particular example these conditions are also necessary. In $[\mathbf{G R}]$ this problem is treated for classical CalderónZygmund operators in $\mathbb{R}^{n}$. There the Riesz transforms are used to show that those classes of weights are necessary. In our setting this rôle will be played by the Cauchy integral. Take a measure $\mu$ for which the Cauchy integral is bounded in $L^{2}(\mu)$, see [To1]. Now, the weighted inequality is

$$
\int_{\mathbb{C}}|\mathcal{C} f(z)|^{p} u(z) d \mu(z) \leq C(u, v) \int_{\mathbb{C}}|f(z)|^{p} v(z) d \mu(z),
$$

for any $f \in L^{p}(v d \mu)$. We devote Section 4 to get the following theorem, which is, essentially, the converse of the previous one.

Theorem. Take p, $1<p<\infty$. Given $0<u<\infty \mu$-a.e. (resp. $0<v<$ $\infty \mu$-a.e.), if there exists some weight $0<v<\infty \mu$-a.e. (resp. $0<u<$ $\infty \mu$-a.e.) such that (2) holds, then $u \in Z_{p}$ (resp. $v \in D_{p}$ ). 
The plan of the paper is the following. Section 2 contains a vectorvalued version of the main theorem in [NTV2], which shall be proved in three steps. In Subsections 2.1 and 2.2 we shall obtain the weak type $(1,1)$ estimate, whereas the strong inequalities are considered in Subsection 2.3. An immediate consequence is given in Subsection 2.4, indeed the vector-valued inequalities obtained there will be the main tool for solving the problem we are concerned with. Sections 3, 4 are devoted to this problem: the first one for general operators and the second one for the particular case of the Cauchy integral, where the necessity is proved.

\section{The vector-valued theorem}

Throughout this section we shall consider vector-valued operators, that is, operators which take their values in Banach spaces.

Let $\mathbb{A}, \mathbb{B}$ be a couple of Banach spaces. $\mathcal{L}(\mathbb{A}, \mathbb{B})$ will denote the set of bounded linear operators from $\mathbb{A}$ to $\mathbb{B}$. We shall say that $K: \mathbb{X} \times \mathbb{X} \longrightarrow$ $\mathcal{L}(\mathbb{A}, \mathbb{B})$ is a (vector-valued) " $n$-dimensional" Calderón-Zygmund kernel if, for some $A>0$, it verifies

(i) $\|K(x, y)\|_{\mathcal{L}(\mathbb{A}, \mathbb{B})} \leq \frac{A}{d(x, y)^{n}}, \quad$ for all $x \neq y$;

(ii) and the following two conditions hold:

$$
\begin{gathered}
\int_{d(x, y) \geq 2 d\left(x, x^{\prime}\right)}\left\|K(x, y)-K\left(x^{\prime}, y\right)\right\|_{\mathcal{L}(\mathbb{A}, \mathbb{B})} d \mu(y) \leq A, \\
\int_{d(x, y) \geq 2 d\left(x, x^{\prime}\right)}\left\|K(y, x)-K\left(y, x^{\prime}\right)\right\|_{\mathcal{L}(\mathbb{A}, \mathbb{B})} d \mu(y) \leq A .
\end{gathered}
$$

Definition 2.1. Let $T$ be a linear operator mapping boundedly $L_{\mathbb{A}}^{2}(\mu)$ into $L_{\mathbb{B}}^{2}(\mu)$, such that, for any $f \in L_{\mathbb{A}}^{2}(\mu)$,

$$
T f(x)=\int_{\mathbb{X}} K(x, y) f(y) d \mu(y), \quad \text { for } \mu \text {-a.e. } x \in \mathbb{X} \backslash \operatorname{supp} f,
$$

where $K$ is an " $n$-dimensional" Calderón-Zygmund kernel. Then we shall say that $T$ is a vector-valued Calderón-Zygmund operator.

For $r>0$, the truncated operators are defined as follows

$$
T_{r} f(x)=\int_{\mathbb{X} \backslash B(x, r)} K(x, y) f(y) d \mu(y),
$$

and we can consider the maximal operator associated with $T$,

$$
T_{\star} f(x)=\sup _{r>0}\left\|T_{r} f(x)\right\|_{\mathbb{B}} .
$$


For $1 \leq p \leq \infty$, it is well known that $L_{\mathbb{A}^{*}}^{p^{\prime}}(\mu) \subset\left(L_{\mathbb{A}}^{p}(\mu)\right)^{*}$. If the Banach space $\mathbb{A}$ is reflexive equality holds, however it fails in general. When we deal with a reflexive Banach space $\mathbb{A}$, we can define $T^{*}$, the adjoint of $T$, which turns out to be a vector-valued CalderónZygmund operator that maps boundedly $L_{\mathbb{B}^{*}}^{2}(\mu)$ into $L_{\mathbb{A}^{*}}^{2}(\mu)$. The kernel is $\widetilde{K}(x, y)=K(y, x)^{*} \in \mathcal{L}\left(\mathbb{B}^{*}, \mathbb{A}^{*}\right)$ (the adjoint operator of $\left.K(y, x)\right)$. Besides, $\left\|T^{*}\right\|_{L_{\mathbb{B}^{*}}^{2}(\mu) \rightarrow L_{\mathrm{A}^{*}}^{2}(\mu)} \leq\|T\|_{L_{\mathbb{A}}^{2}(\mu) \rightarrow L_{\mathbb{B}}^{2}(\mu)}$. Since $\left\|K(y, x)^{*}\right\|_{\mathcal{L}\left(\mathbb{B}^{*}, \mathbb{A}^{*}\right)} \leq$ $\|K(y, x)\|_{\mathcal{L}(\mathbb{A}, \mathbb{B})}$ and

$$
\left\|K\left(y_{1}, x_{1}\right)^{*}-K\left(y_{2}, x_{2}\right)^{*}\right\|_{\mathcal{L}\left(\mathbb{B}^{*}, \mathbb{A}^{*}\right)} \leq\left\|K\left(y_{1}, x_{1}\right)-K\left(y_{2}, x_{2}\right)\right\|_{\mathcal{L}(\mathbb{A}, \mathbb{B})},
$$

$\widetilde{K}$ will be an " $n$-dimensional Calderón-Zygmund kernel with the same constant $A$.

Let $M(\mathbb{X})$ be the space of all complex-valued Borel measures on $\mathbb{X}$. The space $\mathbb{A} \otimes M(\mathbb{X})$ will consist of all finite linear combinations of elements of the form $a \eta$ with $a \in \mathbb{A}$ and $\eta \in M(\mathbb{X})$. For one of these elements we define by convenience

$$
T(a \eta)(x)=\int_{\mathbb{X}} K(x, y) a d \eta(y), \quad x \in \mathbb{X} \backslash \operatorname{supp} \eta .
$$

As in [NTV2], we consider the following version of the Hardy-Littlewood maximal function:

$$
\widetilde{\mathcal{M}} f(x)=\sup _{r>0} \frac{1}{\mu(B(x, 3 r))} \int_{B(x, r)}|f| d \mu .
$$

This maximal function is bounded in $L^{p}(\mu), 1<p \leq \infty$, and acts continuously from $L^{1}(\mu)$ to $L^{1, \infty}(\mu)$.

We shall need the following result, which is a kind of boundedness of an "atom" away from its support.

Lemma 2.2. For $\eta=\sum_{i=1}^{J} a_{i} \eta_{i} \in \mathbb{A} \otimes M(\mathbb{X})$ with $\operatorname{supp} \eta \subset B(x, \rho)$ and

$$
\eta(\mathbb{X})=\int_{\mathbb{X}} d \eta=\sum_{i=1}^{J} a_{i} \int_{\mathbb{X}} d \eta_{i}=\sum_{i=1}^{J} a_{i} \eta_{i}(\mathbb{X})=0,
$$

we have

$$
\int_{\mathbb{X} \backslash B(x, 2 \rho)}\|T \eta(y)\|_{\mathbb{B}} d \mu(y) \leq A \sum_{i=1}^{J}\left\|a_{i}\right\|_{\mathbb{A}}\left\|\eta_{i}\right\|,
$$

where $A$ is the constant in the definition of the kernel. 
Proof: The proof is standard. By using the properties of $\eta$ and condition (ii), we can write

$$
\begin{aligned}
& \int_{\mathbb{X} \backslash B(x, 2 \rho)}\|T \eta(y)\|_{\mathbb{B}} d \mu(y) \\
& =\int_{\mathbb{X} \backslash B(x, 2 \rho)}\left\|\sum_{i=1}^{J} \int_{B(x, \rho)}\left(K\left(y, x^{\prime}\right)-K(y, x)\right) a_{i} d \eta_{i}\left(x^{\prime}\right)\right\|_{\mathbb{B}} d \mu(y) \\
& \leq \sum_{i=1}^{J}\left\|a_{i}\right\|_{\mathbb{A}} \int_{B(x, \rho)} \int_{d(x, y) \geq 2 d\left(x, x^{\prime}\right)}\left\|K\left(y, x^{\prime}\right)-K(y, x)\right\|_{\mathcal{L}(\mathbb{A}, \mathbb{B})} d \mu(y) d\left|\eta_{i}\right|\left(x^{\prime}\right) \\
& \leq A \sum_{i=1}^{J}\left\|a_{i}\right\|_{\mathbb{A}}\left\|\eta_{i}\right\| .
\end{aligned}
$$

Remark 2.3. Just as before, the following can be proved: if $\eta=$ $\sum_{i=1}^{J} a_{i} \eta_{i}+f d \mu \in \mathbb{A} \otimes M(\mathbb{X})+L_{\mathbb{A}}^{1}(\mu)$ with $\operatorname{supp} \eta \subset B(x, \rho)$ and $\eta(\mathbb{X})=\sum_{i=1}^{J} a_{i} \eta_{i}(\mathbb{X})+\int_{\mathbb{X}} f d \mu=0$, we also obtain

$$
\int_{\mathbb{X} \backslash B(x, 2 \rho)}\|T \eta(y)\|_{\mathbb{B}} d \mu(y) \leq A\left(\sum_{i=1}^{J}\left\|a_{i}\right\|_{\mathbb{A}}\left\|\eta_{i}\right\|+\|f\|_{L_{\mathbb{A}}^{1}(\mu)}\right) .
$$

\subsection{Weak type inequality for elementary measures.}

An elementary measure will be an element of $\mathbb{A} \otimes M(\mathbb{X})$, where the measures involved are unit point masses, namely

$$
\nu=\sum_{i=1}^{N} \alpha_{i} \delta_{x_{i}} \in \mathbb{A} \otimes M(\mathbb{X}) .
$$

Theorem 2.4. For an elementary measure as above, the following inequality holds

$$
\|T \nu\|_{L_{\mathbb{B}}^{1, \infty}(\mu)} \leq C \sum_{i=1}^{N}\left\|\alpha_{i}\right\|_{\mathbb{A}}
$$

where $C$ only depends on the dimension $n$, the constant $A$ in the definition of the kernel $K$ and the norm $\|T\|_{L_{\mathbb{A}}^{2}(\mu) \rightarrow L_{\mathbb{B}}^{2}(\mu)}$. 
Observe that here, there is no problem with the definition of $T \nu$ because the sum is finite and

$$
T \nu(x)=\sum_{i=1}^{N} T\left(\alpha_{i} \delta_{x_{i}}\right)(x)=\sum_{i=1}^{N} K\left(x, x_{i}\right) \alpha_{i}
$$

makes sense everywhere except at finitely many points.

Proof: We shall follow the proof of [NTV2, Theorem 5.1] paying special attention to those details that differ from the scalar case. We can assume that $\sum_{i=1}^{N}\left\|\alpha_{i}\right\|_{\mathbb{A}}=1$ and prove that $\|T \nu\|_{L_{\mathbb{B}}^{1, \infty}(\mu)} \leq C$. Fix some $t>0$, and suppose that $\mu(\mathbb{X})>\frac{1}{t}$. Following the "scalar" case proof - with $\left\|\alpha_{i}\right\|_{\mathbb{A}}$ instead of $\alpha_{i}$ - we are able to find some Borel sets $E_{1}, \ldots, E_{N}$ such that

$$
B^{\prime}\left(x_{i}, \rho_{i}\right) \backslash \bigcup_{\ell=1}^{i-1} E_{\ell} \subset E_{i} \subset B\left(x_{i}, \rho_{i}\right) \backslash \bigcup_{\ell=1}^{i-1} E_{\ell} \quad \text { and } \quad \mu\left(E_{i}\right)=\frac{\left\|\alpha_{i}\right\|_{\mathbb{A}}}{t},
$$

where $B^{\prime}\left(x_{i}, \rho_{i}\right)=\left\{y \in \mathbb{X}: d\left(x_{i}, y\right)<\rho_{i}\right\}$. It is clear that the sets $E_{i}$ are pairwise disjoint, if we put $E=\bigcup_{i} E_{i}$,

$$
\bigcup_{i} B^{\prime}\left(x_{i}, \rho_{i}\right) \subset E \subset \bigcup_{i} B\left(x_{i}, \rho_{i}\right) \quad \text { and } \quad \mu(E)=\frac{1}{t} .
$$

Define

$$
\sigma=\sum_{i} \chi_{\mathbb{X} \backslash B\left(x_{i}, 2 \rho_{i}\right)} T\left(\frac{\alpha_{i}}{\left\|\alpha_{i}\right\|_{\mathbb{A}}} \chi_{E_{i}}\right)
$$

and

$$
T \nu-t \sigma=\sum_{i} \varphi_{i}=\sum_{i}\left(T\left(\alpha_{i} \delta_{x_{i}}\right)-t \chi_{\mathbb{X} \backslash B\left(x_{i}, 2 \rho_{i}\right)} T\left(\frac{\alpha_{i}}{\left\|\alpha_{i}\right\|_{\mathbb{A}}} \chi_{E_{i}}\right)\right) .
$$

Since $B^{\prime}\left(x_{i}, \rho_{i}\right) \subset E$, we have

$$
\begin{gathered}
\int_{\mathbb{X} \backslash E}\left\|\varphi_{i}\right\|_{\mathbb{B}} d \mu \leq \int_{\mathbb{X} \backslash B\left(x_{i}, 2 \rho_{i}\right)}\left\|T\left(\alpha_{i} \delta_{x_{i}}-t \frac{\alpha_{i}}{\left\|\alpha_{i}\right\|_{\mathbb{A}}} \chi_{E_{i}} d \mu\right)\right\|_{\mathbb{B}} d \mu \\
+\int_{B\left(x_{i}, 2 \rho_{i}\right) \backslash B^{\prime}\left(x_{i}, \rho_{i}\right)}\left\|T\left(\alpha_{i} \delta_{x_{i}}\right)\right\|_{\mathbb{B}} d \mu \\
\leq 2 A\left\|\alpha_{i}\right\|_{\mathbb{A}}+2^{n} A\left\|\alpha_{i}\right\|_{\mathbb{A}},
\end{gathered}
$$

where we have used Lemma 2.2 for the first term and condition (i) of the kernel for the second. Thus

$$
\int_{\mathbb{X} \backslash E}\|T \nu-t \sigma\|_{\mathbb{B}} d \mu \leq \sum_{i=1}^{N} \int_{\mathbb{X} \backslash E}\left\|\varphi_{i}\right\|_{\mathbb{B}} d \mu \leq 2^{n+1} A \sum_{i=1}^{N}\left\|\alpha_{i}\right\|_{\mathbb{A}}=2^{n+1} A,
$$


and $\mu\left\{x \in \mathbb{X}:\|(T \nu-t \sigma)(x)\|_{\mathbb{B}}>2^{n+1} A t\right\} \leq \frac{2}{t}$, since $\mu(E)=\frac{1}{t}$. Then, it might be enough to find some big constant $A_{0}$ such that

$$
\mu\left\{\|\sigma\|_{\mathbb{B}}>A_{0}\right\} \leq \frac{2}{t} .
$$

In this case, $\mu\left\{x \in \mathbb{X}:\|T \nu(x)\|_{\mathbb{B}}>\left(2^{n+1} A+A_{0}\right) t\right\} \leq \frac{4}{t}$. In order to finish we only have to observe that the above inequality is obvious when $\mu(\mathbb{X}) \leq \frac{1}{t}$. Then, if we take $C=4\left(2^{n+1} A+A_{0}\right)$, we have just obtained $\|T \nu\|_{L_{\mathbb{B}}^{1, \infty}(\mu)} \leq C$.

Let us show how can we get (3) in this vector-valued framework. First, we prove this inequality under the assumption that $\mathbb{A}$ is a reflexive Banach space. For a fixed $A_{0}$, to be chosen later, suppose that $\mu\left\{\|\sigma\|_{\mathbb{B}}>\right.$ $\left.A_{0}\right\}>\frac{2}{t}$. Then, there exists a Borel set $F, F \subset\left\{\|\sigma\|_{\mathbb{B}}>A_{0}\right\}$, such that $\mu(F)=\frac{1}{t}$. Thus $\sigma \chi_{F} \in L_{\mathbb{B}}^{1}(\mu)$, because

$$
\int_{\mathbb{X}}\left\|\sigma \chi_{F}\right\|_{\mathbb{B}} d \mu \leq \mu(F)^{1 / 2}\|\sigma\|_{L_{\mathbb{B}}^{2}(\mu)} \leq\|T\|_{L_{\mathbb{A}}^{2}(\mu) \rightarrow L_{\mathbb{B}}^{2}(\mu)} \frac{1}{t} \sum_{i=1}^{N}\left\|\alpha_{i}\right\|_{\mathbb{A}}^{1 / 2}<\infty .
$$

Since $L_{\mathbb{B}}^{1}(\mu)$ is isometrically contained in $\left(L_{\mathbb{B}^{*}}^{\infty}(\mu)\right)^{*}$, the Hahn-Banach theorem implies the existence of some $\beta \in L_{\mathbb{B}^{*}}^{\infty}(\mu),\|\beta\|_{L_{\mathbb{B}^{*}}^{\infty}(\mu)}=1$, such that

$$
\text { (4) }\left\langle\beta, \sigma \chi_{F}\right\rangle=\left\|\sigma \chi_{F}\right\|_{\left(L_{\mathbb{B}^{*}}^{\infty}(\mu)\right)^{*}}=\int_{F}\|\sigma(x)\|_{\mathbb{B}} d \mu(x)>A_{0} \mu(F)=\frac{A_{0}}{t} \text {. }
$$

On the other hand, $\beta \chi_{F} \in L_{\mathbb{B}^{*}}^{2}(\mu)$ with $\left\|\beta \chi_{F}\right\|_{L_{\mathbb{B}^{*}}^{2}(\mu)} \leq t^{-1 / 2}$ and we can use the adjoint operator to obtain

$$
\begin{aligned}
\left\langle\beta, \sigma \chi_{F}\right\rangle & =\int_{\mathbb{X}}\left\langle\sigma(x) \chi_{F}(x), \beta(x)\right\rangle d \mu(x) \\
& =\sum_{i=1}^{N} \int_{\mathbb{X}}\left\langle\frac{\alpha_{i}}{\left\|\alpha_{i}\right\|_{\mathbb{A}}} \chi_{E_{i}}(x), T^{*}\left(\beta \chi_{F \backslash B\left(x_{i}, 2 \rho_{i}\right)}\right)(x)\right\rangle d \mu(x) \\
& \leq \sum_{i=1}^{N} \int_{\mathbb{X}} \chi_{E_{i}}(x)\left\|T^{*}\left(\beta \chi_{F \backslash B\left(x_{i}, 2 \rho_{i}\right)}\right)(x)\right\|_{\mathbb{A}^{*}} d \mu(x) .
\end{aligned}
$$

For every $x \in E_{i} \subset B\left(x_{i}, \rho_{i}\right)$, by condition (i) of the kernel,

$$
\begin{aligned}
& \left\|T^{*}\left(\beta \chi_{F \backslash B\left(x_{i}, 2 \rho_{i}\right)}\right)(x)-T^{*}\left(\beta \chi_{F \backslash B\left(x, \rho_{i}\right)}\right)(x)\right\|_{\mathbb{A}^{*}} \\
& \quad \leq \int_{B\left(x_{i}, 2 \rho_{i}\right) \backslash B\left(x, \rho_{i}\right)}\|\widetilde{K}(x, y)\|_{\mathcal{L}\left(\mathbb{B}^{*}, \mathbb{A}^{*}\right)}\|\beta(y)\|_{\mathbb{B}^{*}} d \mu(y) \leq 2^{n} A .
\end{aligned}
$$


Hence, if $x \in E_{i}$

$$
\left\|T^{*}\left(\beta \chi_{F \backslash B\left(x_{i}, 2 \rho_{i}\right)}\right)(x)\right\|_{\mathbb{A}^{*}} \leq 2^{n} A+\left(T^{*}\right)_{\star}\left(\beta \chi_{F}\right)(x) .
$$

Then, by the pairwise disjointness of the sets $E_{i}$, we have

$$
\left\langle\beta, \sigma \chi_{F}\right\rangle \leq 2^{n} A \mu(E)+\int_{\mathbb{X}} \chi_{E}(x)\left(T^{*}\right)_{\star}\left(\beta \chi_{F}\right)(x) d \mu(x) .
$$

To finish, we need the following Guy David-type lemma which shall be proved later.

Lemma 2.5. Let $F \subset \mathbb{X}$ be a finite measure Borel set and $\beta \in L_{\mathbb{B}^{*}}^{\infty}$ such that $\|\beta\|_{L_{\mathbb{B}^{*}}}^{\infty}=1$. Then for every $x \in \operatorname{supp} \mu$ we have

$$
\left(T^{*}\right)_{\star}\left(\beta \chi_{F}\right)(x) \leq 2 \cdot 3^{n} \widetilde{\mathcal{M}}\left(\left\|T^{*}\left(\beta \chi_{F}\right)\right\|_{\mathbb{A}^{*}}\right)(x)+A_{1},
$$

where $A_{1}=5 \cdot 3^{n} A+\sqrt{2 \cdot 3^{n}}\|T\|_{L_{\mathbb{A}}^{2}(\mu) \rightarrow L_{\mathbb{B}}^{2}(\mu)}$.

According to this lemma and Hölder's inequality, we can obtain

$$
\begin{aligned}
\int_{\mathbb{X}} \chi_{E}(x) & \left(T^{*}\right)_{\star}\left(\beta \chi_{F}\right)(x) d \mu(x) \\
& \leq A_{1} \mu(E)+2 \cdot 3^{n}\left\|\chi_{E}\right\|_{L^{2}(\mu)}\left\|\widetilde{\mathcal{M}}\left(\left\|T^{*}\left(\beta \chi_{F}\right)\right\|_{\mathbb{A}^{*}}\right)\right\|_{L^{2}(\mu)} \\
& \leq \frac{1}{t}\left(A_{1}+2 \cdot 3^{n}\|\widetilde{\mathcal{M}}\|_{L^{2}(\mu) \rightarrow L^{2}(\mu)}\|T\|_{L_{\mathbb{A}}^{2}(\mu) \rightarrow L_{\mathbb{B}}^{2}(\mu)}\right) .
\end{aligned}
$$

In short, by choosing $A_{0}=2^{n} A+A_{1}+2 \cdot 3^{n}\|\widetilde{\mathcal{M}}\|_{L^{2}(\mu) \rightarrow L^{2}(\mu)}\|T\|_{L_{\AA}^{2}(\mu) \rightarrow L_{\mathbb{B}}^{2}(\mu)}$, we have proved that $\left\langle\beta, \sigma \chi_{F}\right\rangle \leq \frac{A_{0}}{t}$ which contradicts (4); and therefore $\mu\left\{\|\sigma\|>A_{0}\right\} \leq \frac{2}{t}$. When the Banach space $\mathbb{A}$ is not reflexive we proceed as follows. Let $\mathbb{A}_{0}$ be the vectorial subspace of $\mathbb{A}$ generated by $\alpha_{1}, \ldots, \alpha_{N}$, which is a finite-dimensional Banach space and thus reflexive. Set $T_{0}$ the restriction of $T$ to $\mathbb{A}_{0}$-valued functions. The kernel of $T_{0}$ will be $K_{0} \in \mathcal{L}\left(\mathbb{A}_{0}, \mathbb{B}\right)$ (the restriction of $K$ to $\mathbb{A}_{0}$ ) which is clearly a Calderón-Zygmund kernel. In fact, conditions (i) and (ii) are fulfilled by $K_{0}$ with the constant $A$ of $K$. Moreover, $\left\|T_{0}\right\|_{L_{\mathbb{A}_{0}}^{2}(\mu) \rightarrow L_{\mathbb{B}}^{2}(\mu)} \leq$ $\|T\|_{L_{\mathbb{A}}^{2}(\mu) \rightarrow L_{\mathbb{B}}^{2}(\mu)}$, and thus $T_{0}$ is a vector-valued Calderón-Zygmund operator with " $n$-dimensional" kernel $K_{0}$. On the other hand,

$\sigma=\sum_{i} \chi_{\mathbb{X} \backslash B\left(x_{i}, 2 \rho_{i}\right)} T\left(\frac{\alpha_{i}}{\left\|\alpha_{i}\right\|_{\mathbb{A}}} \chi_{E_{i}}\right)=\sum_{i} \chi_{\mathbb{X} \backslash B\left(x_{i}, 2 \rho_{i}\right)} T_{0}\left(\frac{\alpha_{i}}{\left\|\alpha_{i}\right\|_{\mathbb{A}}} \chi_{E_{i}}\right)$.

Lemma 2.5 provides a new constant $A_{1}^{0}$ that verifies

$$
A_{1}^{0}=5 \cdot 3^{n} A+\sqrt{2 \cdot 3^{n}}\left\|T_{0}\right\|_{L_{\mathbb{A}_{0}}^{2}(\mu) \rightarrow L_{\mathbb{B}}^{2}(\mu)} \leq A_{1},
$$


where $A_{1}=5 \cdot 3^{n} A+\sqrt{2 \cdot 3^{n}}\|T\|_{L_{\mathbb{A}}^{2}(\mu) \rightarrow L_{\mathbb{B}}^{2}(\mu)}$ does not depend of $\mathbb{A}_{0}$. Then, as in the previous case, we obtain

$$
\begin{aligned}
\left\langle\beta, \sigma \chi_{F}\right\rangle & \leq \frac{1}{t}\left(2^{n} A+A_{1}^{0}+2 \cdot 3^{n}\|\widetilde{\mathcal{M}}\|_{L^{2}(\mu) \rightarrow L^{2}(\mu)}\left\|T_{0}\right\|_{L_{\mathbb{A}_{0}}^{2}(\mu) \rightarrow L_{\mathbb{B}}^{2}(\mu)}\right) \\
& \leq \frac{1}{t}\left(2^{n} A+A_{1}+2 \cdot 3^{n}\|\widetilde{\mathcal{M}}\|_{L^{2}(\mu) \rightarrow L^{2}(\mu)}\|T\|_{L_{\mathbb{A}}^{2}(\mu) \rightarrow L_{\mathbb{B}}^{2}(\mu)}\right)=\frac{A_{0}}{t},
\end{aligned}
$$

and we get again a contradiction which finishes the non-reflexive case.

Let us prove Lemma 2.5.

Proof of Lemma 2.5: We follow the ideas of [NTV2, Lemma 4.1]. Let $x \in \operatorname{supp} \mu$ and $r>0$. Consider the sequence of balls $B\left(x, r_{j}\right)$ with $r_{j}=3^{j} r$ and set $\mu_{j}=\mu\left(B\left(x, r_{j}\right)\right)$. We can choose $k \geq 1$, the smallest positive integer such that $\mu_{k} \leq 2 \cdot 3^{n} \mu_{k-1}$. Put $R=r_{k-1}=3^{k-1} r$. Then

$$
\begin{aligned}
\| T_{r}^{*}\left(\beta \chi_{F}\right)(x)- & T_{3 R}^{*}\left(\beta \chi_{F}\right)(x) \|_{\mathbb{A}^{*}} \\
& \leq \int_{B(x, 3 R) \backslash B(x, r)}\|\tilde{K}(x, y)\|_{\mathcal{L}\left(\mathbb{B}^{*}, \mathbb{A}^{*}\right)} d \mu(y) \\
& \leq \sum_{j=1}^{k} \int_{B\left(x, r_{j}\right) \backslash B\left(x, r_{j-1}\right)}\|K(y, x)\|_{\mathcal{L}(\mathbb{A}, \mathbb{B})} d \mu(y) \leq 4 \cdot 3^{n} A,
\end{aligned}
$$

just as in [NTV2]. Now we handle $T_{3 R}^{*}\left(\beta \chi_{F}\right)(x)$. Note that here we have the doubling condition $\mu_{k} \leq 2 \cdot 3^{n} \mu_{k-1}$. Define

$$
V_{R}(x)=\frac{1}{\mu(B(x, R))} \int_{B(x, R)} T^{*}\left(\beta \chi_{F}\right) d \mu,
$$

which verifies $\left\|V_{R}(x)\right\|_{\mathbb{A}^{*}} \leq 2 \cdot 3^{n} \widetilde{\mathcal{M}}\left(\left\|T^{*}\left(\beta \chi_{F}\right)\right\|_{\mathbb{A}^{*}}\right)(x)$, because of this doubling condition. On the other hand,

$$
\begin{aligned}
& V_{R}(x)=\frac{1}{\mu(B(x, R))} \int_{B(x, R)} T^{*}\left(\beta \chi_{F \backslash B(x, 3 R)}\right) d \mu \\
& \quad+\frac{1}{\mu(B(x, R))} \int_{B(x, R)} T^{*}\left(\beta \chi_{F \cap B(x, 3 R)}\right) d \mu=I+I I
\end{aligned}
$$


and $\left\|T_{3 R}^{*}\left(\beta \chi_{F}\right)(x)-V_{R}(x)\right\|_{\mathbb{A}^{*}} \leq\left\|T_{3 R}^{*}\left(\beta \chi_{F}\right)(x)-I\right\|_{\mathbb{A}^{*}}+\|I I\|_{\mathbb{A}^{*}}$. By using the second condition on the kernel,

$$
\begin{aligned}
& \left\|T_{3 R}^{*}\left(\beta \chi_{F}\right)(x)-I\right\|_{\mathbb{A}^{*}} \\
& \leq \frac{1}{\mu(B(x, R))} \int_{B(x, R)} \int_{d(x, y) \geq 2 d(x, z)}\|K(y, x)-K(y, z)\|_{\mathcal{L}(\mathbb{A}, \mathbb{B})} d \mu(y) d \mu(z) \\
& \leq \frac{1}{\mu(B(x, R))} \int_{B(x, R)} A d \mu(z)=A .
\end{aligned}
$$

Whereas for the second term, by Hölder's inequality,

$$
\begin{aligned}
\|I I\|_{\mathbb{A}^{*}} & \leq \frac{1}{\mu(B(x, R))^{1 / 2}}\left\|T^{*}\right\|_{L_{\mathbb{B}^{*}}^{2}(\mu) \rightarrow L_{\mathbb{A}^{*}}^{2}(\mu)}\left\|\beta \chi_{F \cap B(x, 3 R)}\right\|_{L_{\mathbb{B}^{*}}^{2}(\mu)} \\
& \leq\left(\frac{\mu(B(x, 3 R))}{\mu(B(x, R))}\right)^{1 / 2}\|T\|_{L_{\AA}^{2}(\mu) \rightarrow L_{\mathbb{B}}^{2}(\mu)} \leq \sqrt{2 \cdot 3^{n}}\|T\|_{L_{\AA}^{2}(\mu) \rightarrow L_{\mathbb{B}}^{2}(\mu)} .
\end{aligned}
$$

Thus, we get $\left\|T_{r}^{*}\left(\beta \chi_{F}\right)(x)\right\|_{\mathbb{A}^{*}} \leq A_{1}+2 \cdot 3^{n} \widetilde{\mathcal{M}}\left(\left\|T^{*}\left(\beta \chi_{F}\right)\right\|_{\mathbb{A}^{*}}\right)(x)$, where $A_{1}=5 \cdot 3^{n} A+\sqrt{2 \cdot 3^{n}}\|T\|_{L_{\mathbb{A}}^{2}(\mu) \rightarrow L_{\mathbb{B}}^{2}(\mu)}$. By taking the supremum on $r>0$ we have the desired estimate.

\subsection{Weak type inequality for functions in $L_{\AA}^{1}(\mu)$.}

Theorem 2.6. Let $f \in L_{\AA}^{1}(\mu) \cap L_{\AA}^{2}(\mu)$, then

$$
\|T f\|_{L_{\mathbb{B}}^{1, \infty}(\mu)} \leq C\|f\|_{L_{\mathbb{A}}^{1}(\mu)},
$$

where $C>0$ only depends on the dimension n, the constant $A$ in the definition of the Calderón-Zygmund kernel $K$ and the norm $\|T\|_{L_{\mathbb{A}}^{2}(\mu) \rightarrow L_{\mathbb{B}}^{2}(\mu)}$.

Proof: Let $C_{0}(\mathbb{X}, \mathbb{A})$ be the space of $\mathbb{A}$-valued functions on $\mathbb{X}$, which are bounded, continuous and with bounded support. It is clear that $C_{0}(\mathbb{X}, \mathbb{A}) \subset L_{\mathbb{A}}^{1}(\mu) \cap L_{\mathbb{A}}^{2}(\mu)$ and this inclusion is dense with respect to the norm $\|\cdot\|_{L_{\AA}^{1}(\mu)}+\|\cdot\|_{L_{\mathbb{A}}^{2}(\mu)}$-in fact, $\mathbb{A} \otimes C_{0}(\mathbb{X})$ is dense in $\mathbb{A} \otimes\left(L^{1}(\mu) \cap\right.$ $\left.L^{2}(\mu)\right)$ which, at the same time, is dense in $\left.L_{\AA}^{1}(\mu) \cap L_{\mathbb{A}}^{2}(\mu)\right)$ - Therefore it is enough to prove the above inequality for functions $f \in C_{0}(\mathbb{X}, \mathbb{A})$. Fix $t>0$ and set $G=\left\{x \in \mathbb{X}:\|f(x)\|_{\mathbb{A}}>t\right\}$. We can split the function $f$ as follows

$$
f=f^{t}+f_{t}=f \chi_{G}+f \chi_{\mathbb{X} \backslash G},
$$


and then $T f=T f^{t}+T f_{t}$. First, we observe that

$\int_{\mathbb{X}}\left\|T f_{t}\right\|_{\mathbb{B}}^{2} d \mu \leq\|T\|_{L_{\mathbb{A}}^{2}(\mu) \rightarrow L_{\mathbb{B}}^{2}(\mu)}^{2} \int_{\mathbb{X}}\left\|f_{t}\right\|_{\mathbb{A}}^{2} d \mu \leq\|T\|_{L_{\mathbb{A}}^{2}(\mu) \rightarrow L_{\mathbb{B}}^{2}(\mu)}^{2} t\|f\|_{L_{\mathbb{A}}^{1}(\mu)}$,

and $\mu\left\{x \in \mathbb{X}:\left\|T f_{t}(x)\right\|_{\mathbb{B}}>t\|T\|_{L_{\AA}^{2}(\mu) \rightarrow L_{\mathbb{B}}^{2}(\mu)}\right\} \leq \frac{1}{t}\|f\|_{L_{\AA}^{1}(\mu)}$. On the other hand, since $G$ is an open set ( $f$ is continuous) and $\mu(G) \leq \frac{1}{t}\|f\|_{L_{\AA}^{1}(\mu)}$, Whitney decomposition can be performed to find a sequence of pairwise disjoint open sets $G_{i}(i=1,2, \ldots)$, such that,

$$
G=\bigcup_{i=1}^{\infty} G_{i} \quad \text { and } \quad \operatorname{diam} G_{i} \leq \frac{1}{2} d\left(G_{i}, \mathbb{X} \backslash G\right) .
$$

Put $f_{i}=f \chi_{G_{i}}$. Then the series $f^{t}=\sum_{i=1}^{\infty} f_{i}$ converges in $L_{\mathbb{A}}^{2}(\mu)$ (due to the fact that the sets are pairwise disjoint and $\left.f \in L_{\AA}^{2}(\mu)\right)$. Set

$$
f^{(N)}=\sum_{i=1}^{N} f_{i} \quad \text { and } \quad \alpha_{i}=\int_{\mathbb{X}} f_{i} d \mu=\int_{G_{i}} f d \mu .
$$

It is clear that $\sum_{i=1}^{\infty}\left\|\alpha_{i}\right\|_{\mathbb{A}} \leq\|f\|_{L_{\mathbb{A}}^{1}(\mu)}$. Choose a point $x_{i}$ in every set $G_{i}$ and consider the elementary measure $\nu_{N}=\sum_{i=1}^{N} \alpha_{i} \delta_{x_{i}}$. Lemma 2.2 and Remark 2.3 lead to

$$
\begin{aligned}
\int_{\mathbb{X} \backslash G}\left\|T f^{(N)}-T \nu_{N}\right\|_{\mathbb{B}} d \mu & \leq \sum_{i=1}^{N} \int_{\mathbb{X} \backslash B\left(x_{i}, 2 \operatorname{diam} G_{i}\right)}\left\|T\left(f_{i} d \mu-\alpha_{i} \delta_{x_{i}}\right)\right\|_{\mathbb{B}} d \mu \\
& \leq \sum_{i=1}^{N} A\left(\left\|f_{i}\right\|_{L_{\mathbb{A}}^{1}(\mu)}+\left\|\alpha_{i}\right\|_{\mathbb{A}}\left\|\delta_{x_{i}}\right\|\right) \leq 2 A\|f\|_{L_{\mathbb{A}}^{1}(\mu)} .
\end{aligned}
$$

Thus $\mu\left\{x \in \mathbb{X} \backslash G:\left\|\left(T f^{(N)}-T \nu_{N}\right)(x)\right\|_{\mathbb{B}}>2 A t\right\} \leq \frac{1}{t}\|f\|_{L_{\mathbb{A}}^{1}(\mu)}$. Moreover, $\nu_{N}$ is an elementary measure and then Theorem 2.4 applies to it with some constant $C_{0}$ in such a way that we get $\mu\{x \in \mathbb{X} \backslash G$ : $\left.\left\|T \nu_{N}(x)\right\|_{\mathbb{B}}>C_{0} t\right\} \leq \frac{1}{t}\|f\|_{L_{\AA}^{1}(\mu)}$. Consequently

$$
\begin{aligned}
& \mu\left\{x \in \mathbb{X}:\left\|T f^{(N)}(x)\right\|_{\mathbb{B}}>\left(2 A+C_{0}\right) t\right\} \\
& \leq \mu(G)+\mu\left\{x \in \mathbb{X} \backslash G:\left\|T f^{(N)}(x)\right\|_{\mathbb{B}}>\left(2 A+C_{0}\right) t\right\} \leq \frac{3}{t}\|f\|_{L_{\mathbb{A}}^{1}(\mu)} .
\end{aligned}
$$


Since $f^{(N)} \longrightarrow f^{t}$ in $L_{\mathbb{A}}^{2}(\mu)$ as $N \rightarrow \infty$, we have $T f^{(N)} \longrightarrow T f^{t}$ in $L_{\mathbb{B}}^{2}(\mu)$ as $N \rightarrow \infty$. Then

$$
\mu\left\{x \in \mathbb{X}:\left\|T f^{t}(x)\right\|_{\mathbb{B}}>\left(2 A+C_{0}\right) t\right\} \leq \frac{3}{t}\|f\|_{L_{\mathbb{A}}^{1}(\mu)}
$$

and the desired inequality holds with $C=4\left(\|T\|_{L_{\mathbb{A}}^{2}(\mu) \rightarrow L_{\mathbb{B}}^{2}(\mu)}+2 A+\right.$ $C_{0}$ ).

\subsection{Strong inequalities.}

Theorem 2.7. The operator $T$ is continuous from $L_{\mathbb{A}}^{p}(\mu)$ to $L_{\mathbb{B}}^{p}(\mu)$ for all $p, 1<p<\infty$.

Proof: For $1<p \leq 2$, it is enough to apply the Marcinkiewicz interpolation theorem. In the other case, $2 \leq p<\infty$, assume that $\mathbb{A}$ is a reflexive Banach space. Then the adjoint operator $T^{*}$ will be a vector-valued Calderón-Zygmund operator. By using the previous case, $T^{*}$ is bounded between $L_{\mathbb{B}^{*}}^{p^{\prime}}(\mu)$ and $L_{\mathbb{A}^{*}}^{p^{\prime}}(\mu)$. Thus, $T$ is continuous from $L_{\mathbb{A}}^{p}(\mu)$ to $L_{\mathbb{B}}^{p}(\mu)$. When $\mathbb{A}$ is a non-reflexive Banach space, consider $\mathbb{A}_{0}$ any finitedimensional subspace of $\mathbb{A}$. Let $T_{0}$ be the restriction of $T$ to $\mathbb{A}_{0}$-valued functions. As we observed in the proof of Theorem 2.4, $T_{0}$ is a vectorvalued Calderón-Zygmund operator for which all the constants involved are independent of the chosen subspace. Since $\mathbb{A}_{0}$ is reflexive, by the reasoning above, we obtain that $T_{0}$ is bounded from $L_{\mathbb{A}_{0}}^{p}(\mu)$ to $L_{\mathbb{B}}^{p}(\mu)$ with a constant independent of $\mathbb{A}_{0}$. On the other hand, $\mathbb{A} \otimes L^{p}(\mu)$ is dense in $L_{\AA}^{p}(\mu)$, and any element $f \in \mathbb{A} \otimes L^{p}(\mu)$, has an expression $f=\sum_{i=1}^{m} \alpha_{i} f_{i}$, for some $\alpha_{1}, \ldots, \alpha_{m} \in \mathbb{A}$ and $f_{1}, \ldots, f_{m} \in L^{p}(\mu)$. By calling $\mathbb{A}_{0}$ to the finite-dimensional subspace generated by $\alpha_{1}, \ldots, \alpha_{m}$, we have that $f \in L_{\mathbb{A}_{0}}^{p}(\mu)$ and

$$
\|T f\|_{L_{\mathbb{B}}^{p}(\mu)}=\left\|T_{0} f\right\|_{L_{\mathbb{B}}^{p}(\mu)} \leq C\|f\|_{L_{\mathrm{A}_{0}}^{p}(\mu)}=C\|f\|_{L_{\mathbb{A}}^{p}(\mu)},
$$

where $C$ is independent of $\mathbb{A}_{0}$.

Remark 2.8. The previous results still hold if we replace the a priori continuity of $T$ from $L_{\mathbb{A}}^{2}(\mu)$ to $L_{\mathbb{B}}^{2}(\mu)$ by the assumption that $T$ is bounded between $L_{\mathbb{A}}^{p_{0}}(\mu)$ and $L_{\mathbb{B}}^{p_{0}}(\mu)$, for some other $p_{0}$, with $1<p_{0}<\infty$.

\subsection{Self-improvement.}

Theorems 2.6 and 2.7 have an immediate self-improvement in the following sense: under the same hypotheses we can use these results to extend the operators to sequence-valued functions. 
Theorem 2.9. Let $T$ be a vector-valued Calderón-Zygmund operator and take $q, 1<q<\infty$. Then

(i) $T$ is bounded from $L_{\ell_{\mathbb{A}}^{q}}^{1}(\mu)$ to $L_{\ell_{\mathbb{B}}^{q}}^{1, \infty}(\mu)$, that is,

$$
\mu\left\{x:\left\{\sum_{j}\left\|T f_{j}(x)\right\|_{\mathbb{B}}^{q}\right\}^{\frac{1}{q}}>\lambda\right\} \leq \frac{C}{\lambda} \int_{\mathbb{X}}\left\{\sum_{j}\left\|f_{j}(x)\right\|_{\mathbb{A}}^{q}\right\}^{\frac{1}{q}} d \mu(x) .
$$

(ii) $T$ is bounded from $L_{\ell_{\mathbb{A}}^{q}}^{p}(\mu)$ to $L_{\ell_{\mathbb{B}}^{q}}^{p}(\mu)$, for $1<p<\infty$, that is,

$$
\left\|\left\{\sum_{j}\left\|T f_{j}\right\|_{\mathbb{B}}^{q}\right\}^{\frac{1}{q}}\right\|_{L^{p}(\mu)} \leq C\left\|\left\{\sum_{j}\left\|f_{j}\right\|_{\mathbb{A}}^{q}\right\}^{\frac{1}{q}}\right\|_{L^{p}(\mu)} .
$$

Proof: For $f=\left\{f_{j}\right\}_{j} \in L_{\ell_{\mathbb{A}}^{q}}^{q}(\mu)$ with compact support, we define

$\widetilde{T} f(x)=\left\{T f_{j}(x)\right\}_{j}=\left\{\int_{\mathbb{X}} K(x, y) f_{j}(y) d \mu(y)\right\}_{j}=\int_{\mathbb{X}} \widetilde{K}(x, y) f(y) d \mu(y)$,

for $\mu$-a.e. $x \in \mathbb{X} \backslash \operatorname{supp} f$, where the kernel $\widetilde{K}: \mathbb{X} \times \mathbb{X} \longrightarrow \mathcal{L}\left(\ell_{\mathbb{A}}^{q}, \ell_{\mathbb{B}}^{q}\right)$ is defined by

$$
\widetilde{K}(x, y) \alpha=\left\{K(x, y) \alpha_{j}\right\}_{j}, \quad \text { for any } \alpha=\left\{\alpha_{j}\right\}_{j} \in \ell_{\mathbb{A}}^{q} .
$$

We take the Banach spaces $\widetilde{\mathbb{A}}=\ell_{\mathbb{A}}^{q}$ and $\widetilde{\mathbb{B}}=\ell_{\mathbb{B}}^{q}$. Theorem 2.7 leads to

$$
\|\widetilde{T} f\|_{L_{\ell_{\mathbb{B}}^{q}}^{q}(\mu)}^{q}=\sum_{j} \int_{\mathbb{X}}\left\|T f_{j}\right\|_{\mathbb{B}}^{q} d \mu \leq C \sum_{j} \int_{\mathbb{X}}\left\|f_{j}\right\|_{\mathbb{A}}^{q} d \mu=C\|f\|_{L_{\ell_{\mathbb{A}}^{q}}^{q}(\mu)}^{q} .
$$

The fact that $\widetilde{K}$ is a vector-valued " $n$-dimensional" kernel follows from its expression, because $\|\widetilde{K}(x, y)\|_{\mathcal{L}\left(\ell_{\mathbb{A}}^{q}, \ell_{\mathbb{B}}^{q}\right)} \leq\|K(x, y)\|_{\mathcal{L}(\mathbb{A}, \mathbb{B})}$ and

$$
\left\|\widetilde{K}(x, y)-\widetilde{K}\left(x^{\prime}, y^{\prime}\right)\right\|_{\mathcal{L}\left(\ell_{\mathbb{A}}^{q}, \ell_{\mathbb{B}}^{q}\right)} \leq\left\|K(x, y)-K\left(x^{\prime}, y^{\prime}\right)\right\|_{\mathcal{L}(\mathbb{A}, \mathbb{B})} .
$$

Indeed, the constant $A$ of $K$ is still valid for $\widetilde{K}$. Thus, $\widetilde{T}$ is a vectorvalued Calderón-Zygmund operator —where the a priori estimate holds for $p_{0}=q$ instead of 2 , which is all right because of Remark 2.8 and we can use Theorems 2.6 and 2.7 in order to obtain the desired inequalities.

As a consequence of this result, we can extend those operators considered in [NTV2] to sequence spaces. Namely, let $T$ be a ("scalar") Calderón-Zygmund operator (see Definition 1.1). Here, "scalar" means that the Banach spaces are $\mathbb{A}=\mathbb{B}=\mathbb{C}$. In particular, $T$ fits into the 
vector-valued theory, and we can use the self-improvement result (Theorem 2.9) in order to obtain this sequence-valued extension.

Corollary 2.10. Let $T$ be an operator as above and take $q, 1<q<\infty$. Then

(i) $\mu\left\{x:\left\{\sum_{j}\left|T f_{j}(x)\right|^{q}\right\}^{\frac{1}{q}}>\lambda\right\} \leq \frac{C}{\lambda} \int_{\mathbb{X}}\left\{\sum_{j}\left|f_{j}(x)\right|^{q}\right\}^{\frac{1}{q}} d \mu(x)$.

(ii) $\left\|\left\{\sum_{j}\left|T f_{j}\right|^{q}\right\}^{\frac{1}{q}}\right\|_{L^{p}(\mu)} \leq C\left\|\left\{\sum_{j}\left|f_{j}\right|^{q}\right\}^{\frac{1}{q}}\right\|_{L^{p}(\mu)}$, if $1<p<\infty$.

Remark 2.11. These vector-valued results will be further used in $[\mathbf{G M}]$ to obtain similar estimates for the maximal operator associated with $T$, which, under the appropriate conditions, fits into this vector-valued theory. In particular, we shall prove the previous inequalities for the supremum of the truncated Cauchy integrals. By means of them, weighted inequalities for this maximal operator will be obtained and we shall be able to study the existence of principal values in weighted Lebesgue spaces.

\section{Vector-valued inequalities and weights}

The relation between weighted inequalities and vector-valued inequalities was discovered by J. L. Rubio de Francia in $[\mathbf{R}]$ and it can be also found in Chapter VI of $[\mathbf{G R}]$. The two-weight problem for an operator $T$ consists in finding all pairs $(u, v)$ of positive functions for which the inequality

$$
\int_{\mathbb{X}}|T f(x)|^{p} u(x) d \mu(x) \leq C(u, v) \int_{\mathbb{X}}|f(x)|^{p} v(x) d \mu(x),
$$

$\left(f \in L^{p}(v)\right)$ holds true. We are going to consider the following weak variant of this general problem:

Find conditions on $0 \leq v<\infty \mu$-a.e. (resp. $u>0$ $\mu$-a.e.) such that (5) is satisfied by some $u>0 \mu$-a.e. (resp. $0 \leq v<\infty \mu$-a.e.).

To start, we need the following result, proved in $[\mathbf{F T}]$, which establishes the concrete relationship between vector-valued inequalities and weights. This theorem is closely related to those contained in $[\mathbf{G R}$, pp. 549-554]. 
Theorem 3.1. Let $(\mathbb{Y}, d \nu)$ be a measure space; $\mathbb{F}, \mathbb{G}$ Banach spaces, and $\left\{A_{k}\right\}_{k \in \mathbb{Z}}$ a sequence of pairwise disjoint measurable subsets of $\mathbb{Y}$ such that $\mathbb{Y}=\bigcup_{k} A_{k}$. Consider $0<s<p<\infty$ and $T$ a sublinear operator which satisfies the following vector-valued inequality

$$
\left\|\left\{\sum_{j}\left\|T f_{j}\right\|_{\mathbb{G}}^{p}\right\}^{\frac{1}{p}}\right\|_{L^{s}\left(A_{k}, d \nu\right)} \leq C_{k}\left\{\sum_{j}\left\|f_{j}\right\|_{\mathbb{F}}^{p}\right\}^{\frac{1}{p}}, \quad k \in \mathbb{Z},
$$

where, for every $k \in \mathbb{Z}, C_{k}$ only depends on $\mathbb{F}, \mathbb{G}, p$ and $s$. Then, there exists a positive function $u(x)$ on $\mathbb{Y}$ such that

$$
\left\{\int_{\mathbb{Y}}\|T f(x)\|_{\mathbb{G}}^{p} u(x) d \nu(x)\right\}^{\frac{1}{p}} \leq C\|f\|_{\mathbb{F}}
$$

where $C$ depends on $\mathbb{F}, \mathbb{G}, p$ and $s$. Moreover, given a sequence of positive numbers $\left\{a_{k}\right\}_{k \in \mathbb{Z}}$ with $\sum_{k} a_{k}^{p}<\infty$, and $\sigma=\left(\frac{p}{s}\right)^{\prime}, u(x)$ can be found in such a way that $\left\|u^{-1} \chi_{A_{k}}\right\|_{L^{\sigma-1}\left(A_{k}, d \mu\right)} \leq\left(a_{k}^{-1} C_{k}\right)^{p}$.

In our context $(\mathbb{Y}, d \nu)=(\mathbb{X}, d \mu)$ which is a $\sigma$-finite measure space. Then, a simple argument shows that the weight $u$ can be also taken so that $u<\infty$ a.e.

Given $1<p<\infty$ and some $x_{0} \in \mathbb{X}$, remember the definition of the classes of weights in $\mathbb{X}$ :

$$
\begin{aligned}
& D_{p}=\left\{0 \leq w<\infty \mu \text {-a.e. }: \int_{\mathbb{X}} w(x)^{1-p^{\prime}}\left(1+d\left(x, x_{0}\right)\right)^{-n p^{\prime}} d \mu(x)<\infty\right\} \\
& Z_{p}=\left\{w>0 \text { p-a.e. }: \int_{\mathbb{X}} w(x)\left(1+d\left(x, x_{0}\right)\right)^{-n p} d \mu(x)<\infty\right\} .
\end{aligned}
$$

Note that these classes of weights do not depend on the point $x_{0}$.

Remark 3.2. In the case that the diameter of the space is finite, (or equivalently, the distance is bounded), there exists $R$ large enough such that $\mathbb{X} \subset B\left(x_{0}, R\right)$ and so $\mu(\mathbb{X}) \leq R^{n}<\infty$. Thus, the previous classes can be given by the equivalent definition:

$$
\begin{aligned}
& D_{p}=\left\{0 \leq w<\infty \mu \text {-a.e. }: \int_{\mathbb{X}} w(x)^{1-p^{\prime}} d \mu(x)<\infty\right\} \\
& Z_{p}=\left\{w>0 \mu \text {-a.e. }: \int_{\mathbb{X}} w(x) d \mu(x)<\infty\right\} .
\end{aligned}
$$


If the support of the measure is a bounded set, we can restrict the whole space to this set, and we would be in the previous case. So, when we talk about spaces with finite diameter, we shall be concerned with both cases.

We would like to apply the last theorem to our operators. In what follows $T$ will be a "scalar" Calderón-Zygmund operator $T$, that is, an operator like those in Definition 1.1.

Proposition 3.3. Take $0<s<1<p<\infty$ and $v \in D_{p}$. Then, if the diameter of $\mathbb{X}$ is equal to infinity, we have

$$
\begin{array}{r}
\left\|\left\{\sum_{j}\left|T f_{j}\right|^{p}\right\}^{\frac{1}{p}}\right\|_{L^{s}\left(S_{k}, d \mu\right)} \leq C_{s, p} 2^{\frac{k n}{s}}\left\{\sum_{j}\left\|f_{j}\right\|_{L^{p}(v d \mu)}^{p}\right\}^{\frac{1}{p}}, \\
\quad \text { for } k=0,1, \ldots
\end{array}
$$

where $S_{0}=\left\{x: d\left(x, x_{0}\right) \leq 1\right\}$ and $S_{k}=\left\{x: 2^{k-1}<d\left(x, x_{0}\right) \leq 2^{k}\right\}$, for $k=1,2, \ldots$. Otherwise,

$$
\left\|\left\{\sum_{j}\left|T f_{j}\right|^{p}\right\}^{\frac{1}{p}}\right\|_{L^{s}(\mu)} \leq C_{s, p}\left\{\sum_{j}\left\|f_{j}\right\|_{L^{p}(v d \mu)}^{p}\right\}^{\frac{1}{p}} .
$$

Proof: Let us see what happens in the first situation. Fix $k \geq 0$ and set $B_{k+1}=B\left(x_{0}, 2^{k+1}\right)$. Every function $f$ is split as $f=f^{\prime}+f^{\prime \prime}=$ $f \chi_{B_{k+1}}+f \chi_{\mathbb{X} \backslash B_{k+1}}$. Then, for $x \in S_{k}$ and $y \in \mathbb{X} \backslash B_{k+1}$ we observe that $2 d(x, y)>d\left(y, x_{0}\right)$ and thus

$$
\begin{aligned}
\left|T f^{\prime \prime}(x)\right| & \leq \int_{\mathbb{X} \backslash B_{k+1}} \frac{A}{d(x, y)^{n}}|f(y)| d \mu(y) \\
& \leq 4^{n} A \int_{\mathbb{X}}\left(1+d\left(y, x_{0}\right)\right)^{-n}|f(y)| v(y)^{\frac{1}{p}} v(y)^{-\frac{1}{p}} d \mu(y) \\
& \leq 4^{n} A\left\{\int_{\mathbb{X}}|f(y)|^{p} v(y) d \mu(y)\right\}^{\frac{1}{p}}\left\{\int_{\mathbb{X}} \frac{v(y)^{1-p^{\prime}}}{\left(1+d\left(y, x_{0}\right)\right)^{n p^{\prime}}} d \mu(y)\right\}^{\frac{1}{p^{\prime}}} \\
& \leq C\|f\|_{L^{p}(v d \mu) .}
\end{aligned}
$$


Note that the last inequality holds because $v \in D_{p}$. Then, since $\mu\left(S_{k}\right) \leq$ $\mu\left(B_{k}\right) \leq 2^{k n}$, we prove

$$
\left\|\left\{\sum_{j}\left|T f_{j}^{\prime \prime}\right|^{p}\right\}^{\frac{1}{p}}\right\|_{L^{s}\left(S_{k}, d \mu\right)} \leq C 2^{\frac{k n}{s}}\left\{\sum_{j}\left\|f_{j}\right\|_{L^{p}(v d \mu)}^{p}\right\}^{\frac{1}{p}} .
$$

On the other hand, due to that fact that $0<s<1$, we can use Kolmogorov inequality (see [GR, p. 485]) and Corollary 2.10 to obtain

$$
\begin{aligned}
& \left\|\left\{\sum_{j}\left|T f_{j}^{\prime}\right|^{p}\right\}^{\frac{1}{p}}\right\|_{L^{s}\left(S_{k}, d \mu\right)} \leq C_{s} \mu\left(S_{k}\right)^{\frac{1}{s}-1}\left\|\left\{\sum_{j}\left|T f_{j}^{\prime}\right|^{p}\right\}^{\frac{1}{p}}\right\|_{L^{1, \infty}\left(S_{k}, d \mu\right)} \\
& \leq C \mu\left(S_{k}\right)^{\frac{1}{s}-1} \int_{B_{k+1}}\left\{\sum_{j}\left|f_{j}(x)\right|^{p}\right\}^{\frac{1}{p}} v(x)^{\frac{1}{p}} v(x)^{-\frac{1}{p}} d \mu(x) \\
& \leq C \mu\left(S_{k}\right)^{\frac{1}{s}-1}\left\{\int_{\mathbb{X}} \sum_{j}\left|f_{j}(x)\right|^{p} v(x) d \mu(x)\right\}^{\frac{1}{p}}\left\{\int_{B_{k+1}} v(x)^{-\frac{p^{\prime}}{p}} d \mu(x)\right\}^{\frac{1}{p^{\prime}}} \\
& =C \mu\left(S_{k}\right)^{\frac{1}{s}-1}\left\{\sum_{j}\left\|f_{j}\right\|_{L^{p}(v d \mu)}^{p}\right\}^{\frac{1}{p}}\left\{\int_{B_{k+1}} v(x)^{1-p^{\prime}} d \mu(x)\right\}^{\frac{1}{p^{\prime}}} .
\end{aligned}
$$

As $\frac{1}{s}-1>0$, we observe $\mu\left(S_{k}\right)^{\frac{1}{s}-1} \leq \mu\left(B_{k}\right)^{\frac{1}{s}-1} \leq\left(2^{k n}\right)^{\frac{1}{s}-1}$. Furthermore,

$$
\begin{aligned}
& \left\{\int_{B_{k+1}} v(x)^{1-p^{\prime}} d \mu(x)\right\}^{\frac{1}{p^{\prime}}} \\
= & \left\{\int_{B_{k+1}} \frac{v(x)^{1-p^{\prime}}}{\left(1+d\left(x, x_{0}\right)\right)^{n p^{\prime}}}\left(1+d\left(x, x_{0}\right)\right)^{n p^{\prime}} d \mu(x)\right\}^{\frac{1}{p^{\prime}}} \leq C 2^{n} 2^{(k+1) n},
\end{aligned}
$$

since $v \in D_{p}$. Then,

$$
\left\|\left\{\sum_{j}\left|T f_{j}^{\prime}\right|^{p}\right\}^{\frac{1}{p}}\right\|_{L^{s}\left(S_{k}, d \mu\right)} \leq C 2^{\frac{k n}{s}}\left\{\sum_{j}\left\|f_{j}\right\|_{L^{p}(v d \mu)}^{p}\right\}^{\frac{1}{p}} .
$$

Collecting these inequalities, we get the desired estimate. 
When the space has finite diameter, it measure will be finite as well. Thus, we proceed like we did with the functions $f_{j}^{\prime}$. Since $0<s<1$, we can apply Kolmogorov inequality (see [GR, p. 485]) and Corollary 2.10 to obtain

$$
\begin{aligned}
& \left\|\left\{\sum_{j}\left|T f_{j}\right|^{p}\right\}^{\frac{1}{p}}\right\|\left\|_{L^{s}(\mu)} \leq C_{s} \mu(\mathbb{X})^{\frac{1}{s}-1}\right\|\left\{\sum_{j}\left|T f_{j}\right|^{p}\right\}^{\frac{1}{p}} \|_{L^{1, \infty}(\mu)} \\
& \leq C \mu(\mathbb{X})^{\frac{1}{s}-1} \int_{\mathbb{X}}\left\{\sum_{j}\left|f_{j}(x)\right|^{p}\right\}^{\frac{1}{p}} v(x)^{\frac{1}{p}} v(x)^{-\frac{1}{p}} d \mu(x) \\
& \leq C \mu(\mathbb{X})^{\frac{1}{s}-1}\left\{\int_{\mathbb{X}} \sum_{j}\left|f_{j}(x)\right|^{p} v(x) d \mu(x)\right\}^{\frac{1}{p}}\left\{\int_{\mathbb{X}} v(x)^{-\frac{p^{\prime}}{p}} d \mu(x)\right\}^{\frac{1}{p^{\prime}}} \\
& \leq C\left\{\sum_{j}\left\|f_{j}\right\|_{L^{p}(v d \mu)}^{p}\right\}^{\frac{1}{p}},
\end{aligned}
$$

because $\mathbb{X}$ has finite measure and $v \in D_{p}$ (which, in this case, means $\left.v^{1-p^{\prime}} \in L^{1}(\mu)\right)$.

Once we have the vector-valued inequalities we can use Theorem 3.1 to obtain weighted inequalities.

Theorem 3.4. Take $p, 1<p<\infty$. If $u \in Z_{p}$ (resp. $v \in D_{p}$ ), then there exists some weight $0<v<\infty \mu$-a.e. (resp. $0<u<\infty \mu$-a.e.) such that (5) holds. Moreover, $v$ (resp. u) can be found in such a way that $v^{\alpha} \in Z_{p}$ (resp. $u^{\alpha} \in D_{p}$ ), provided that $0<\alpha<1$.

Proof: Assume that the case $v \in D_{p}$ is proved. If $u \in Z_{p}$, then $\tilde{u}=$ $u^{1-p^{\prime}} \in D_{p^{\prime}}$. Apply this assumption to the adjoint operator $T^{*}$ (which is an operator with the same properties as $T$ ) in order to obtain some weight $\widetilde{v}, 0<\widetilde{v}<\infty \mu$-a.e., such that

$$
\int_{\mathbb{X}}\left|T^{*} f(x)\right|^{p^{\prime}} \widetilde{v}(x) d \mu(x) \leq C \int_{\mathbb{X}}|f(x)|^{p^{\prime}} \widetilde{u}(x) d \mu(x) .
$$

Take $v$ so that $\widetilde{v}=v^{1-p^{\prime}}$. Then, since $0<v<\infty \mu$-a.e., an standard argument yields that the last inequality implies (5). Furthermore, we can choose $\widetilde{v}$ such that $\widetilde{v}^{\alpha} \in D_{p^{\prime}}$, provided that $0<\alpha<1$. That is, we can find $v$ in such a way that $v^{\alpha} \in Z_{p}$. 
Let us prove the case $v \in D_{p}$. Fix $0<\alpha<1$ and put $q=1+\alpha\left(p^{\prime}-1\right)$. Then $1<q<p^{\prime}$ and we can find some $s, 0<s<1$, such that $\sigma=\left(\frac{p}{s}\right)^{\prime}>$ $q$.

When $\mathbb{X}$ has infinite diameter, we use Theorem 3.1 with $(\mathbb{Y}, d \nu)=$ $(\mathbb{X}, d \mu), \mathbb{F}=L^{p}(v d \mu), \mathbb{G}=\mathbb{C},\left\{A_{k}\right\}_{k}=\left\{S_{k}\right\}_{k=0}^{\infty}$ and $C_{k}=C 2^{\frac{k n}{s}}$. The vector-valued inequality (6) is supplied by Proposition 3.3. Then, we know that there exists a weight $u$ such that (5) holds. Moreover, $u$ can be taken in such a way that $\left\|u^{-1}\right\|_{L^{\sigma-1}\left(S_{k}, d \mu\right)} \leq C\left(a_{k}^{-1} 2^{\frac{k n}{s}}\right)^{p}$, with $a_{k}>0$ and $\sum_{k} a_{k}^{p}<\infty$. Therefore,

$$
\begin{gathered}
\int_{\mathbb{X}} \frac{u(x)^{1-q}}{\left(1+d\left(x, x_{0}\right)\right)^{n p^{\prime}}} d \mu(x)=\sum_{k=0}^{\infty} \int_{S_{k}} \frac{u(x)^{1-q}}{\left(1+d\left(x, x_{0}\right)\right)^{n p^{\prime}}} d \mu(x) \\
\leq 2^{n p^{\prime}} \sum_{k=0}^{\infty} 2^{-k n p^{\prime}}\left\{\int_{S_{k}} u(x)^{1-\sigma} d \mu(x)\right\}^{\frac{q-1}{\sigma-1}} \mu\left(S_{k}\right)^{\frac{1}{\left(\frac{\sigma-1}{q-1}\right)^{\prime}}} \\
\leq 2^{n p^{\prime}} C \sum_{k=0}^{\infty} a_{k}^{-p(q-1)} 2^{k n\left(-p^{\prime}+\frac{p(q-1)}{s}+\frac{1}{\left(\frac{\sigma-1}{q-1}\right)^{\prime}}\right)}
\end{gathered}
$$

where we have used Hölder's inequality with exponent $\frac{\sigma-1}{q-1}>1$. Observe that

$$
-p^{\prime}+\frac{p(q-1)}{s}+\frac{1}{\left(\frac{\sigma-1}{q-1}\right)^{\prime}}=q-p^{\prime}<0,
$$

so, we can choose $\varepsilon>0$ such that $q-p^{\prime}+\varepsilon<0$. Take the sequence $\left\{a_{k}\right\}_{k}$ verifying $a_{k}^{-p(q-1)}=2^{k n \varepsilon}$. Then

$$
\sum_{k=0}^{\infty} a_{k}^{p}=\sum_{k=0}^{\infty} 2^{-\frac{k n \varepsilon}{q-1}}<\infty
$$

and

$$
\int_{\mathbb{X}} \frac{u(x)^{1-q}}{\left(1+d\left(x, x_{0}\right)\right)^{n p^{\prime}}} d \mu(x) \leq C \sum_{k=0}^{\infty} 2^{k n\left(q-p^{\prime}+\varepsilon\right)}<\infty .
$$

In order to finish it is enough to note that $\alpha=\frac{1-q}{1-p^{\prime}}$ and thus $u^{\alpha} \in D_{p}$.

When the space has finite diameter, as well as before, we use Theorem 3.1 with $(\mathbb{Y}, d \nu)=(\mathbb{X}, d \mu), \mathbb{F}=L^{p}(v d \mu), \mathbb{G}=\mathbb{C}$. In this case, we do not decompose the space, that is, we just take $A_{0}=\mathbb{X}$ and $A_{k}=\emptyset$ if $k \neq 0$. The vector-valued inequality (6) is provided by the second part of Proposition 3.3. Then, there exists a weight $u$ such that (5) holds. 
Furthermore, $u$ can be taken in such a way that $\left\|u^{-1}\right\|_{L^{\sigma-1}(\mathbb{X}, d \mu)} \leq C$. Since the measure of $\mathbb{X}$ is finite and $\frac{\sigma-1}{q-1}>1$, we use Hölder's inequality for this exponent to conclude

$$
\int_{\mathbb{X}} u(x)^{1-q} d \mu(x) \leq\left\{\int_{\mathbb{X}} u(x)^{1-\sigma} d \mu(x)\right\}^{\frac{q-1}{\sigma-1}} \mu(\mathbb{X})^{\frac{1}{\left(\frac{\sigma-1}{q-1}\right)^{\prime}}<\infty .}
$$

Observe that $\alpha=\frac{1-q}{1-p^{\prime}}$ and we have $u^{\alpha} \in D_{p}$.

\section{Cauchy integral operator}

For a non-negative Borel measure $\mu$ in the complex plane $\mathbb{C}$, the Cauchy integral operator of a compactly supported function $f \in L^{p}(\mu)$, $1 \leq p \leq \infty$, is defined as

$$
\mathcal{C} f(z)=\mathcal{C}_{\mu} f(z)=\int_{\mathbb{C}} \frac{f(\xi)}{z-\xi} d \mu(\xi), \quad \text { for } \mu \text {-a.e. } z \in \mathbb{C} \backslash \operatorname{supp} f .
$$

Assume that $\mu$ is such that the truncated Cauchy integrals are uniformly bounded in $L^{2}(\mu)$. By [To1], $\mu$ will be in particular "1-dimensional". In that case, we know that the existence of the principal value for compactly supported functions holds (see [To2] ). Then, a bounded extension to the whole $L^{2}(\mu)$ arises from these facts. Thus, we have a metric space $\mathbb{C}$ with the euclidean metric and $\mu$ a "1-dimensional" measure for which the Cauchy integral operator is bounded in $L^{2}(\mu)$. We observe that this operator falls into the theory developed by [NTV2]. The Cauchy integral operator is defined for compactly supported function in $L^{2}(\mu)$ by means of its kernel $K(z, \xi)=\frac{1}{z-\xi}$, that is clearly a "1-dimensional" CalderónZygmund kernel. Then we can apply the results we have obtained to get vector-valued inequalities for $\mathcal{C}$. By Corollary 2.10, the following result is established.

Theorem 4.1. Under the above assumptions and for $1<p, q<\infty$ we have

(i) $\mu\left\{z \in \mathbb{C}:\left\{\sum_{j}\left|\mathcal{C} f_{j}(z)\right|^{q}\right\}^{\frac{1}{q}}>\lambda\right\} \leq \frac{C}{\lambda} \int_{\mathbb{C}}\left\{\sum_{j}\left|f_{j}(z)\right|^{q}\right\}^{\frac{1}{q}} d \mu(z)$.

(ii) $\left\|\left\{\sum_{j}\left|\mathcal{C} f_{j}\right|^{q}\right\}^{\frac{1}{q}}\right\|_{L^{p}(\mu)} \leq C\left\|\left\{\sum_{j}\left|f_{j}\right|^{q}\right\}^{\frac{1}{q}}\right\|_{L^{p}(\mu)}$. 
In this framework, for $1<p<\infty$, the classes of weights will be

$$
\begin{aligned}
D_{p} & =\left\{0 \leq w<\infty \mu \text {-a.e. : } \int_{\mathbb{C}} w(z)^{1-p^{\prime}}(1+|z|)^{-p^{\prime}} d \mu(z)<\infty\right\} \\
Z_{p} & =\left\{w>0 \mu \text {-a.e. : } \int_{\mathbb{C}} w(z)(1+|z|)^{-p} d \mu(z)<\infty\right\} .
\end{aligned}
$$

If the measure has bounded support, these classes admit the equivalent definition given in Remark 3.2. In fact, several results will be easier when it happens. For $w \geq 0$ a.e. we denote $w(A)=\int_{A} w(z) d \mu(z)$, for any measurable set $A \subset \mathbb{C}$.

We would like to apply to this operator the results about weights we have proved. The point is that here we can obtain that these classes are sharp for this weak variant of the two-weight problem for the Cauchy integral operator: suppose that for some fixed $0<u, v<\infty \mu$-a.e., the following two-weight inequality holds

$$
\int_{\mathbb{C}}|\mathcal{C} f(z)|^{p} u(z) d \mu(z) \leq C(u, v) \int_{\mathbb{C}}|f(z)|^{p} v(z) d \mu(z),
$$

for any $f \in L^{p}(v d \mu)$. We are going to prove that, in this case, the weights belong to the given classes.

If $z=z_{1}+i z_{2}, \xi=\xi_{1}+i \xi_{2}$ and $f$ is a real-valued function, for $\mu$-a.e. $z \in \mathbb{C} \backslash \operatorname{supp} f$, we observe

$$
\begin{aligned}
\mathcal{C} f(z) & =\operatorname{Re}(\mathcal{C} f(z))+i \operatorname{Im}(\mathcal{C} f(z)) \\
& =\int_{\mathbb{C}} \frac{z_{1}-\xi_{1}}{|z-\xi|^{2}} f(\xi) d \mu(\xi)-i \int_{\mathbb{C}} \frac{z_{2}-\xi_{2}}{|z-\xi|^{2}} f(\xi) d \mu(\xi) .
\end{aligned}
$$

Lemma 4.2. Assume that (7) holds. Then for any $z \in \operatorname{supp} \mu$ there exits a radius $r_{z}>0$, such that, $u\left(B\left(z, r_{z}\right)\right)<\infty$.

Proof: Fix $z_{0}=z_{1}^{0}+i z_{2}^{0} \in \operatorname{supp} \mu$, then $\mu\left(B\left(z_{0}, r\right)\right)>0$ for all $r>0$. For $z=z_{1}+i z_{2}$, we write $|z|_{\infty}=\max \left\{\left|z_{1}\right|,\left|z_{2}\right|\right\}$ and

$F_{1}=\left\{z \in \mathbb{C}:\left|z-z_{0}\right|_{\infty}=z_{1}-z_{1}^{0}\right\}, \quad F_{2}=\left\{z \in \mathbb{C}:\left|z-z_{0}\right|_{\infty}=z_{2}-z_{2}^{0}\right\}$, $F_{3}=\left\{z \in \mathbb{C}:\left|z-z_{0}\right|_{\infty}=z_{1}^{0}-z_{1}\right\}, \quad F_{4}=\left\{z \in \mathbb{C}:\left|z-z_{0}\right|_{\infty}=z_{2}^{0}-z_{2}\right\}$.

Set $B_{k}=B\left(z_{0}, 2^{-k}\right)$ and $S_{k}=B_{k} \backslash B_{k+1}$. Then, there is some $k_{0} \geq 0$ such that $S_{k_{0}}$ has positive measure (otherwise $\mu\left(B_{0}\right)=0$ ). Assume for instance that $\mu\left(S_{k_{0}} \cap F_{1}\right)>0$ (in the other cases we proceed in a similar way). Thus, there will exist $A \subset S_{k_{0}} \cap F_{1}$ so that $\mu(A)>0$ and $v(A)<\infty$. For $z \in B_{k_{0}+2}$ and $\xi \in A$, we have $|z-\xi| \leq 5 \cdot 2^{-k_{0}-2}$. Since 
$\xi \in A \subset F_{1}$,

$$
2^{-k_{0}-1} \leq\left|\xi-z_{0}\right| \leq \sqrt{2} \max \left\{\left|\xi_{1}-z_{1}^{0}\right|,\left|\xi_{2}-z_{2}^{0}\right|\right\}=\sqrt{2}\left(\xi_{1}-z_{1}^{0}\right) .
$$

Besides, $z_{1}-z_{1}^{0} \geq-\left|z-z_{0}\right| \geq-2^{-k_{0}-2}$ and

$$
\xi_{1}-z_{1}=\xi_{1}-z_{1}^{0}+z_{1}^{0}-z_{1} \geq \frac{1}{\sqrt{2}}\left|\xi-z_{0}\right|-2^{-k_{0}-2} \geq(\sqrt{2}-1) 2^{-k_{0}-2} .
$$

Therefore, for $z \in B_{k_{0}+2}, \xi \in A$

$$
\frac{\xi_{1}-z_{1}}{|z-\xi|^{2}} \geq \frac{(\sqrt{2}-1) 2^{-k_{0}-2}}{\left(5 \cdot 2^{-k_{0}-2}\right)^{2}}=\frac{\sqrt{2}-1}{25} 2^{k_{0}+2}=C_{k_{0}} .
$$

Then, if $z \in B_{k_{0}+2}$,

$$
-\operatorname{Re}\left(\mathcal{C}\left(\chi_{A}\right)(z)\right)=\int_{A} \frac{\xi_{1}-z_{1}}{|z-\xi|^{2}} d \mu(\xi) \geq C_{k_{0}} \mu(A)=C>0 .
$$

So, for the left hand side of (7) we have

$$
\begin{aligned}
\int_{\mathbb{C}}\left|\mathcal{C}\left(\chi_{A}\right)(z)\right|^{p} u(z) d \mu(z) & \geq \int_{B_{k_{0}+2}}\left(-\operatorname{Re}\left(\mathcal{C}\left(\chi_{A}\right)(z)\right)\right)^{p} u(z) d \mu(z) \\
& \geq C^{p} \int_{B_{k_{0}+2}} u(z) d \mu(z) .
\end{aligned}
$$

Use this estimate and (7), with $f=\chi_{A} \in L^{p}(v)$, to obtain $u\left(B_{k_{0}+2}\right)<$ $\infty$. Then, by taking $r_{z_{0}}=2^{-k_{0}-2}$ the proof is finished.

Lemma 4.3. Assume that (7) holds, then there exists $R>0$ such that

$$
\int_{\mathbb{C} \backslash B(0, R)} \frac{u(z)}{(1+|z|)^{p}} d \mu(z)<\infty .
$$

Proof: For $j=1, \ldots, 4$, set $E_{j}$ by putting $z_{0}=0$ in the definition of $F_{j}$. Then, it might be enough to find some $R_{j}>0$, for each $j$, such that,

$$
\int_{E_{j} \backslash B\left(0, R_{j}\right)} \frac{u(z)}{(1+|z|)^{p}} d \mu(z)<\infty .
$$

We shall only do it for $j=1$ and the other cases can be performed in the same manner. We can assume that $\mu\left(E_{1}\right)>0$ (otherwise there is nothing to prove). If $E \bigcap \operatorname{supp} \mu$ is a bounded set, the estimate is trivial by choosing $R_{1}$ large enough. In the other case, there exists $R_{1}$ such that $\mu\left(B\left(0, R_{1} / 2\right) \bigcap E_{1}\right)>0$. Take $A \subset B\left(0, R_{1} / 2\right) \cap E_{1}$ with $\mu(A)>0$ and $v(A)<\infty$. Then, for $z \in E_{1} \backslash B\left(0, R_{1}\right)$ and $\xi \in A,|z|>2|\xi|$ and $|z-\xi| \leq|z|+|\xi| \leq \frac{3}{2}|z|$. Moreover, since both points belong to $E_{1}$,

$$
|z|=\sqrt{z_{1}^{2}+z_{2}^{2}} \leq \sqrt{2} \max \left\{\left|z_{1}\right|,\left|z_{2}\right|\right\}=\sqrt{2} z_{1}, \quad \xi_{1}=\left|\xi_{1}\right| \leq|\xi|<\frac{1}{2}|z|,
$$


and hence

$$
z_{1}-\xi_{1} \geq \frac{1}{\sqrt{2}}|z|-\frac{1}{2}|z|=\frac{\sqrt{2}-1}{2}|z| .
$$

Then,

$$
\frac{z_{1}-\xi_{1}}{|z-\xi|^{2}} \geq \frac{2(\sqrt{2}-1)}{9} \frac{1}{|z|} \geq \frac{2(\sqrt{2}-1)}{9} \frac{1}{1+|z|},
$$

and for $z \in E_{1} \backslash B\left(0, R_{1}\right)$,

$$
\begin{aligned}
\operatorname{Re}\left(\mathcal{C}\left(\chi_{A}\right)(z)\right) & =\int_{A} \frac{z_{1}-\xi_{1}}{|z-\xi|^{2}} d \mu(\xi) \geq \frac{2(\sqrt{2}-1)}{9} \frac{1}{1+|z|} \mu(A) \\
& =\frac{C}{1+|z|}>0 .
\end{aligned}
$$

Therefore, for the left hand side of (7) we get

$$
\begin{aligned}
\int_{\mathbb{C}}\left|\mathcal{C}\left(\chi_{A}\right)(z)\right|^{p} u(z) d \mu(z) & \geq \int_{E_{1} \backslash B\left(0, R_{1}\right)}\left(\operatorname{Re}\left(\mathcal{C}\left(\chi_{A}\right)(z)\right)\right)^{p} u(z) d \mu(z) \\
& \geq C^{p} \int_{E_{1} \backslash B\left(0, R_{1}\right)} \frac{u(z)}{(1+|z|)^{p}} d \mu(z) .
\end{aligned}
$$

Since $v(A)<\infty,(7)$ can be used. Then the right hand side of this inequality is finite and the proof is finished.

Now, we are able to prove the following result, which, together with Theorem 3.4, gives us necessary and sufficient conditions on the weights in order to solve, for the Cauchy integral operator, the weak variant of the two-weight problem we are dealing with.

Theorem 4.4. Take $p, 1<p<\infty$. Given $0<u<\infty \mu$-a.e. (resp. $0<$ $v<\infty \mu$-a.e.), if there exists some weight $0<v<\infty \mu$-a.e. (resp. $0<$ $u<\infty \mu$-a.e.) such that (7) holds, then $u \in Z_{p}$ (resp. $\left.v \in D_{p}\right)$.

Proof: We shall use the previous lemmas. Fix $0<u, v<\infty \mu$-a.e. such that ( 7 ) holds. By taking the radius $R>0$ supplied by Lemma 4.3 , we only have to see what happens on the ball. Lemma 4.2 and a compactness argument lead to

$$
\int_{B(0, R)} \frac{u(z)}{(1+|z|)^{p}} d \mu(z)<\infty .
$$


Thus $u \in Z_{p}$. In order to show that $v \in D_{p}$, we shall use a duality argument. By decomposing the space in the dyadic level sets where $2^{j} \leq v(z)<2^{j+1}$ and since $0<v<\infty$ a.e., we can prove that boundedly supported functions in $L^{p}(\mu) \cap L^{p}\left(v^{-1}\right) \cap L^{\infty}(\mu)$ are dense in $L^{p}(\mu)$. This fact allows us to obtain that (7) implies

$$
\int_{\mathbb{C}}|\mathcal{C} f(z)|^{p^{\prime}} v(z)^{1-p^{\prime}} d \mu(z) \leq C(u, v) \int_{\mathbb{C}}|f(z)|^{p^{\prime}} u(z)^{1-p^{\prime}} d \mu(z) .
$$

By taking into account what we have just proved, we obtain $v^{1-p^{\prime}} \in Z_{p^{\prime}}$, that is, $v \in D_{p}$.

Remark 4.5. In [GR, pp. 560-562], similar results are proved for classical Calderón-Zygmund operators in $\mathbb{R}^{n}$. There, necessary and sufficient conditions on the weights are obtained for the Riesz transforms. Those ideas are not valid here for general measures. If we had that $\mu\left(E_{i}\right)>0$, for all $i=1, \ldots, 4$, the proof of the previous theorem would be simpler by following that method. However, in general, we are not guaranteed to have this property. That is the reason why we have used Lemmas 4.2 and 4.3 .

\section{References}

[BCP] A. Benedek, A.-P. Calderón and R. Panzone, Convolution operators on Banach space valued functions, Proc. Nat. Acad. Sci. U.S.A. 48 (1962), 356-365.

[Cal] A.-P. CALderón, Cauchy integrals on Lipschitz curves and related operators, Proc. Nat. Acad. Sci. U.S.A. 74(4) (1977), 1324-1327.

[Ch1] M. ChRIST, "Lectures on singular integral operators", CBMS Regional Conference Series in Mathematics 77, published for the Conference Board of the Mathematical Sciences, Washington, DC, 1990.

[Ch2] M. Christ, A $T(b)$ theorem with remarks on analytic capacity and the Cauchy integral, Colloq. Math. 60/61(2) (1990), 601-628.

[CMM] R. R. Coifman, A. Mcintosh and Y. Meyer, L'intégrale de Cauchy définit un opérateur borné sur $L^{2}$ pour les courbes lipschitziennes, Ann. of Math. (2) 116(2) (1982), 361-387.

[Da1] G. DAVID, "Wavelets and singular integrals on curves and surfaces", Lecture Notes in Mathematics 1465, Springer-Verlag, Berlin, 1991. 
[Da2] G. David, Analytic capacity, Calderón-Zygmund operators, and rectifiability, Publ. Mat. 43(1) (1999), 3-25.

[DJS] G. David, J.-L. Journé And S. Semmes, Opérateurs de Calderón-Zygmund, fonctions para-accrétives et interpolation, Rev. Mat. Iberoamericana 1(4) (1985), 1-56.

[FT] L. M. FÉrnandez-Cabrera and J. L. Torrea, Vectorvalued inequalities with weights, Publ. Mat. 37(1) (1993), 177-208.

[GM] J. García-Cuerva and J. M. Martell, On the existence of principal values for the Cauchy integral on weighted Lebesgue spaces for non-doubling measures, Preprint (1999).

[GR] J. García-Cuerva and J. L. Rubio de Francia, "Weighted norm inequalities and related topics", North-Holland Mathematics Studies 116, North-Holland Publishing Co., Amsterdam, 1985.

[MMV] P. Mattila, M. S. Melnikov and J. Verdera, The Cauchy integral, analytic capacity, and uniform rectifiability, Ann. of Math. (2) 144(1) (1996), 127-136.

[Mel] M. S. Melnikov, Analytic capacity: a discrete approach and the curvature of measure, Mat. Sb. 186(6) (1995), 57-76.

[MV] M. S. Melnikov And J. Verdera, A geometric proof of the $L^{2}$ boundedness of the Cauchy integral on Lipschitz graphs, Internat. Math. Res. Notices 7 (1995) 325-331.

[Mur] T. MuraI, "A real variable method for the Cauchy transform, and analytic capacity", Lecture Notes in Mathematics 1307, Springer-Verlag, Berlin, 1988.

[NTV1] F. Nazarov, S. Treil and A. Volberg, Cauchy integral and Calderón-Zygmund operators on nonhomogeneous spaces, Internat. Math. Res. Notices 15 (1997), 703-726.

[NTV2] F. Nazarov, S. Treil and A. Volberg, Weak type estimates and Cotlar inequalities for Calderón-Zygmund operators on nonhomogeneous spaces, Internat. Math. Res. Notices 9 (1998), 463-487.

[OP] J. Orobitg and C. PÉrez, $A_{p}$ weights for non-doubling measures in $\mathbb{R}^{n}$ and applications, Preprint (1999).

[R] J. L. Rubio De Francia, Weighted norm inequalities and vector-valued inequalities, in: "Harmonic analysis" (Minneapolis, Minn., 1981), Lecture Notes in Mathematics 908, SpringerVerlag, Berlin, 1982, pp. 86-101. 
[RRT] J. L. Rubio de Francia, F. J. Ruiz and J. L. Torrea, Calderón-Zygmund theory for operator-valued kernels, Adv. in Math. 62(1) (1986), 7-48.

[Ste] E. M. Stein, "Harmonic analysis: real-variable methods, orthogonality, and oscillatory integrals, Princeton Mathematical Series 43, Princeton University Press, Princeton, NJ, 1993.

[To1] X. ToLSA, $L^{2}$-boundedness of the Cauchy integral operator for continuous measures, Duke Math. J. 98(2) (1999), 269-304.

[To2] X. TOLSA, Cotlar's inequality without the doubling condition and existence of principal values for the Cauchy integral of measures, J. Reine Angew. Math. 502 (1998), 199-235.

[Ver] J. Verdera, On the T(1) theorem for the Cauchy integral, Ark. Mat. 38(1) (2000), 183-199.

Departamento de Matemáticas, C-XV

Universidad Autónoma de Madrid

28049 Madrid

Spain

E-mail address: jose.garcia-cuerva@uam.es

E-mail address: chema.martelll@uam.es

Primera versió rebuda el 28 de gener de 2000, darrera versió rebuda el 14 d'abril de 2000. 